\title{
Partial Discharge Simulation under Various Applied Voltage Waveforms
}

\author{
Hazlee Illias, Low Tau Jian, Ab Halim Abu Bakar, Hazlie Mokhlis \\ Electrical Engineering Department, UMPEDAC \\ Faculty of Engineering, University of Malaya \\ Kuala Lumpur, Malaysia \\ h.illias@um.edu.my
}

\begin{abstract}
In high voltage power equipment, partial discharge (PD) measurement is widely used in the performance assessment of an insulation system. However, from modelling of PD, a better understanding of the phenomenon may be attained. PD activity within a void in a solid dielectric insulation material is influenced by many factors. One of the factors is the applied voltage. Different types of the applied voltage yield in different PD patterns and the condition for breakdown of insulation to occur. In this work, PD activity under various types of the applied voltages has been studied. A finite element analysis (FEA) software was used to simulate PD occurrence within a void in a dielectric material. The applied voltages that were considered are AC sinusoidal, damped AC and impulse voltages. PD patterns were observed through phase resolved partial discharge (PRPD) patterns. The obtained result may improve an understanding on PD behaviour under different types of the applied voltage. It may also assist in insulation diagnostics and performance assessment of an insulation system.
\end{abstract}

Keywords-partial discharge; condition monitoring; finite element analysis; electrical insulation

\section{INTRODUCTION}

In high voltage equipment, the build-up of charge and its by-products can be symptomatic of problems associated with aging and insulation breakdown. If such problems are not repaired, the strength and frequency of partial discharge (PD) increase, which eventually leads to the catastrophic failure of the high voltage equipment [1]. This may cause external equipment damage, resulting in a high repair cost due to an unscheduled outage. Therefore, a reliable online PD detection is important and is a must for power companies to improve the safety and decrease the potential of plant shut down. This is one of the reasons why PD detection is used in power systems to monitor the condition of high voltage equipment.

One of the factors which influences PD activity within insulation system of high voltage equipment is the applied voltage waveform. PD activity within a spherical void in a dielectric material under $\mathrm{AC}$ sinusoidal applied voltage has been studied through measurement and simulation [2-5]. The results found that at higher frequency and amplitude of the applied voltage, the number of PDs per cycle and the total PD charge per cycle increase. PD patterns were found to follow the waveform of the sinusoidal voltage. There were two distinguished patterns formed, a 'rabbit-ear' and 'tortoise like' patterns. These patterns were due to the difference in the difficulty of electron detrapping from the void surface with different polarity of charges accumulated on it. Simulation of PD was performed and compared to the measurement results. From comparison, critical parameters influencing PD activity under AC sinusoidal applied voltage have been successfully identified.

PD mechanism in a silicone gel has been studied through experiment under $\mathrm{AC}$ sinusoidal and impulse voltages in a point plane geometry [6]. Under impulse applied voltage, PD behaviour shows that the first discharges recorded in a silicon gel were due to the initiation and propagation of streamers. However, under AC applied voltage, a stable $\mathrm{PD}$ regime rapidly establishes, where a streamer creates a void with a long lifetime and PDs occur in the void during the subsequent voltage cycle. This resulted in permanent degradations of the gel due to the repetition of PDs. These findings clearly indicate that PD mechanism is strongly influenced by the applied voltage conditions.

In this paper, PD activity within a void in a dielectric material under various types of the applied voltages has been studied through simulation. A PD model and finite element analysis (FEA) software were used to simulate PD events. Phase resolved partial discharge (PRPD) patterns were used to observe PD patterns. The applied voltages that were simulated are $\mathrm{AC}$ sinusoidal, damped $\mathrm{AC}$ and impulse voltages. From the study of PD events under different applied voltages, PD activity can be characterised more precisely and critical parameters affecting PD activity under different types of applied voltage can be identified. This may assist in performance assessment and diagnostics of insulation system.

\section{PARTIAL Discharge MODEL EQUATIONS}

The first condition for a PD to occur within a void in a dielectric material is the electric field in the void, $E_{\text {void }}$ must be larger than the inception field, $E_{\text {inc. }} E_{\text {inc }}$ is calculated using [7]

$$
E_{\text {inc }}=25.2 P\left[1+8.6 /(2 \mathrm{Pr})^{0.5}\right]
$$

where $P$ and $r$ are the pressure and radius of the void. 
The second condition for a PD to occur is a starting electron is needed to start ionization. The supply of an initial electron influences the statistical characteristics of PD activity such as the inception delay, the number of PD occurrences and distributions with respect to the applied voltage. In a virgin void, a starting electron may be provided by natural high energetic radiation via photo ionization in the gas or by photo ionization in the insulation. The starting electrons can also be produced by field emission from the void surface if its effective work function is low $[7,8]$.

For a void which has never experienced PD, volume ionization is the main source of an initial free electron. If the void consists of air, the rate of volume ionization can be calculated using $[7,8]$

$$
N_{v}=20 P\left(\pi a b^{2}\right)\left(1-v^{-2}\right)
$$

where $a$ and $b$ are the height and radius of the void. $v$ is the ratio of the applied voltage to the inception voltage or the overvoltage ratio [7],

$$
v=U_{0} / U_{\text {oinc }}
$$

where $U_{0}$ is the maximum applied voltage and $U_{\text {oinc }}$ is the inception voltage.

After the first PD has occurred, an additional source of initial electron becomes available through charges along the void surface from the previous PD. A free initial electron emitted from the void surface, $\dot{N}_{d t}$ can be expressed as [7]

$$
\dot{N}_{d t}=N_{d t} v_{o} \exp \left(-\left[\phi-\sqrt{ }\left[e E_{\text {void }} /\left(4 \pi \varepsilon_{0}\right)\right] /(k T)\right]\right)
$$

where $\dot{N}_{d t}$ is the number of electrons available for being detrapped, $v_{o}$ is the fundamental phonon frequency $\left(\approx 10^{14} \mathrm{~Hz}\right)$, $e$ is the elementary charge, $\Phi$ is the effective detrapping work function, $\varepsilon_{0}$ is the permittivity of vacuum, $k$ is the Boltzmann constant and $T$ is the temperature (in Kelvin). $N_{d t}$ is the detrappable electrons from the surface due to previous PD, which decays between consecutive PDs. It has been defined as [7]

$$
N_{d t}=N_{d t 0} \exp (-t / \tau)
$$

where $t$ is the time elapsed since the last PD event and $\tau$ is the effective charge decay time constant. $N_{d t}$ has been assumed to be proportional to the number of charges, $q$ due to $\mathrm{PD}$ [7],

$$
\begin{aligned}
& N_{d t 0}=\xi(q / e) \\
& q=\varepsilon_{0} \pi b^{2}\left[1+\varepsilon_{r}(K-1)\right] \Delta E
\end{aligned}
$$

where $\xi$ is the proportionality factor, $b$ is the void radius, $\varepsilon_{r}$ is the material permittivity, $\Delta E$ is the field collapse in the void after a PD occurs and $K$ equals to 3 for a spherical void. $\Delta E$ is the electric field change in the void after a PD has occurred. It is calculated using

$$
\Delta E=E_{\text {voidnoPD }}+E_{q}-E_{\text {ext }}
$$

where $E_{\text {voidnoPD }}$ is the field in the void in the absence of PD, $E_{q}$ is the field due to charges on the void surface after a PD has occurred and $E_{\text {ext }}$ is the extinction field.

The proportionality factor, $\xi$ depends on the polarity of the surface charge from which electron detrapping occurs. This is because the negative and positive charges on the void surface have a different efficiency of allowing electrons to detrap form the void surface [7]. An electron detraps from negative surface charges after the polarity of $E_{\text {void }}$ changes between consecutive PDs but not when the surface charges are negative.

The total number of electrons, $N_{t}$ available to initiate a PD is equal to

$$
N_{t}=\dot{N}_{d t}+N_{v}
$$

A PD occurrence is a random process. Thus, the probability, $P$ of a PD to occur is defined as $N_{t} \Delta t$, where $\Delta t$ is the time step of the simulation. A PD will only occur if $P$ is larger than a random number, $R$, which is between 0 and 1 .

The dipolar charges due to the PD induce charges on the conductors on the insulation material. These charges are called the apparent charge, $q^{\prime}[7,8]$. For a plane-parallel electrode with a gap distance of $D$ and void radius of $a, q$ ' equals to

$$
q^{\prime}=(4 / 3) \pi a b^{2} \varepsilon_{0} \varepsilon_{r} K \Delta E / D
$$

\section{MODEL GEOMETRY}

A two-dimensional (2D) axial-symmetric model geometry consists of a spherical void and a cylindrical dielectric material has been developed using finite element analysis (FEA) software, as shown in Figure 1. The model has been used to obtain the electric field in the void, $E_{\text {voidnoPD }}$ in the absence of PD through simulation of the electric field distribution in the model. The obtained value was used in (8) for the applied voltage of $19.25 \mathrm{kV}$. The parameters used for the simulation are summarised in Table 1 and were chosen based on the literature [7].

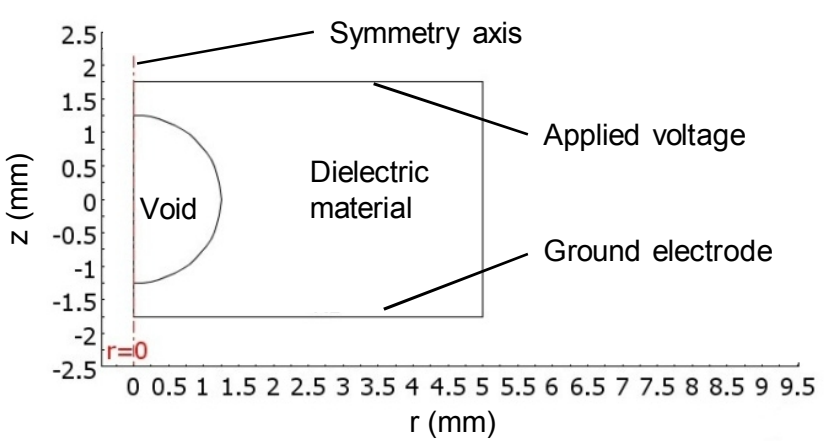

Figure 1. 2D axial-symmetric model geometry 
TABLE I. BOUNDARY CONDITIONS OF THE MODEL

\begin{tabular}{|c|c|c|}
\hline Parameter Description & Symbol & Value \\
\hline Spherical void radius & $r, \mathrm{a}, b$ & $1.25 \mathrm{~mm}$ \\
\hline Dielectric material thickness & $D$ & $3.50 \mathrm{~mm}$ \\
\hline Dielectric material permittivity & $\varepsilon_{r m a t}$ & 4 \\
\hline Void permittivity & $\varepsilon_{r v o i d}$ & 1 \\
\hline Applied voltage & $U_{a p p}$ & $19.25 \mathrm{kV}$ \\
\hline Dielectric material radius & $r_{\text {mat }}$ & $5.00 \mathrm{~mm}$ \\
\hline Void pressure & $P$ & $65 \mathrm{kPa}$ \\
\hline Temperature & $T$ & $300 \mathrm{~K}$ \\
\hline Time step & $\Delta t$ & $0.0001 \mathrm{~s}$ \\
\hline Over voltage ratio & $v$ & 3.5 \\
\hline Proportionality factor & $\zeta$ & 50 \\
\hline Material surface work function & $\Phi$ & $1.1 \mathrm{eV}$ \\
\hline Effective charge decay time constant & $\tau$ & $2 \mathrm{~ms}$ \\
\hline Number of simulation cycles & & 200 \\
\hline
\end{tabular}

\section{SimUlation RESUlts}

\section{A. PD under AC sinusoidal applied voltage}

Figure 2 shows the electric field magnitude as a function of time of $50 \mathrm{~Hz}, 19.25 \mathrm{kV}$ sinusoidal applied voltage obtained using FEA and PD model equations for the first two cycles. When the electric field within the void, $E_{\text {void }}$ exceeds the inception field, $E_{i n c}$ and if there is a free initial electron available, a PD will occur, causing $E_{\text {void }}$ to drop less than the extinction field, $E_{\text {ext }}$. If there is no PD occurs at all, $E_{\text {void }}$ will follow the curve of $E_{\text {voidnoPD }}$, which is the electric field in the void without any surface charge due to $\mathrm{PD}$. At the time between $0.005 \mathrm{~s}$ and $0.01 \mathrm{~s}$, PD does not occur immediately when $E_{\text {void }}$ is higher than the inception field there is no free initial electron available for a PD to occur. This delay is called the statistical time lag, $\tau_{\text {stat }}$.

For PD activity under AC sinusoidal voltage, there are two different types of statistical time lag. The first type is $\tau_{\text {stat }}$ due to the first PD. This time lag is long because electrons are harder to be emitted from the void surface due to the absence of electron accumulating on the void surface. Electrons are likely to be available from volume ionisation. The second type of $\tau_{\text {stat }}$ is $\tau_{\text {stat }}$ after the previous PD has occurred. This time lag is shorter because there are more free electrons available on the void surface after the previous PD has occurred. The statistical time lag influences the number of PDs per cycle.

One of the factors which influences the PD activity within a void in a dielectric material is the effective charge decay time constant, $\tau$. To study the effect of this parameter, the material surface work function, $\Phi$ is fixed at $1.1 \mathrm{eV}$ but $\tau$ is varied from $1 \mathrm{~ms}$ to $3 \mathrm{~ms}$ in (5). The phase resolved partial discharge (PRPD) patterns are used to observe PD patterns under different parameters of the simulation and conditions of the applied voltage.

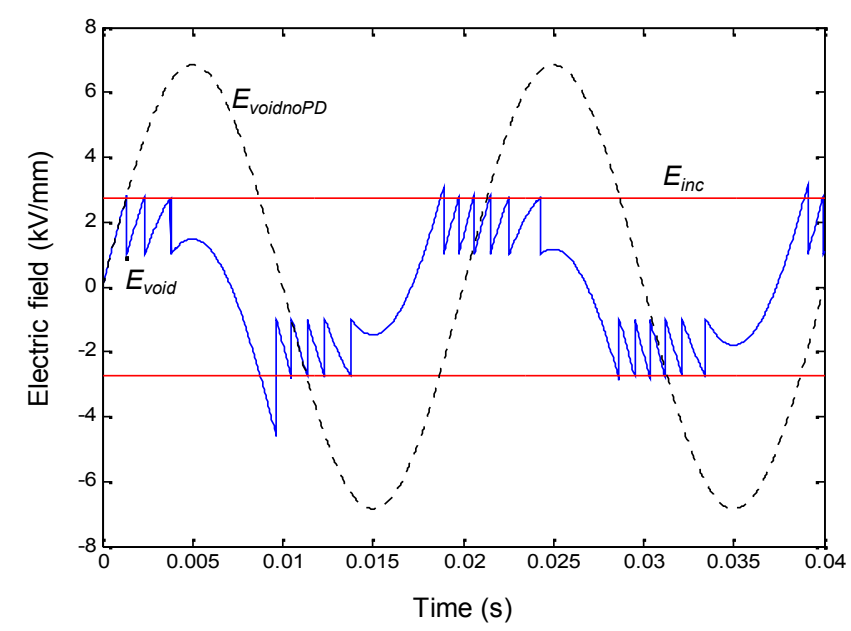

Figure 2. Electric field magnitude against time of sinusoidal applied voltage

A PRPD pattern shows the occurrence of PDs at a specific phase of the applied voltage (in degree) with a certain charge magnitude (in $\mathrm{nC}$ ), as represented by the 'dots' in Figure 3 . In a PRPD pattern, PDs occurring in certain number of applied voltage cycle are grouped together into one applied voltage cycle, ranging from 0 to 360 degrees. If more PDs occur within an applied voltage cycle, more 'dot' can be seen in a PRPD pattern. PRPD patterns are commonly used in condition monitoring of high voltage equipment to observe PD activity.

From Figure 3, the PRPD patterns change significantly when $\tau$ is varied. When $\tau$ is increased, the number of PDs per cycle, $N_{P D}$ increases. $\tau$ controls the rate of charges that have accumulated on the void surface due to previous PD to decay with time. From (4), when $\tau$ is larger, the electron detrapping due to surface emission, $\dot{N}_{d t}$ becomes higher. This is due to a longer $\tau$ causes the accumulated charges on the void surface due to previous PD to decay slower with time. Thus, the total number of electrons, $N_{t}$ available to initiate the next PD is higher, resulting in a higher probability of a PD to occur when the inception field, $E_{i n c}$ in the void is exceeded. This results in PD to occur almost immediately after $E_{i n c}$ has been exceeded, yielding the maximum PD charge magnitude to decrease with longer $\tau$ and more PDs occurring at lower PD charge magnitudes. A lower charge magnitude is obtained when a PD occurs at a lower electric field in the void.

\section{B. Damped AC sinusoidal applied voltage}

The mathematical expression of a damped AC sinusoidal applied voltage waveform can be represented by the product of an $\mathrm{AC}$ sinusoidal waveform and the inverse of exponential,

$$
U=U_{a p p} \sin (2 \pi f t) e^{-t / \tau_{d}}
$$

where $f$ is the frequency, $t$ is the time and $\tau_{d}$ is the decay constant of the waveform. To study the effect of damped AC sinusoidal voltage on PD activity within a void in a dielectric material, the applied frequency is set at $50 \mathrm{~Hz}$ while $\tau_{d}$ is varied from 0.25 to 4 . All simulation parameters are shown in Table 1 . 


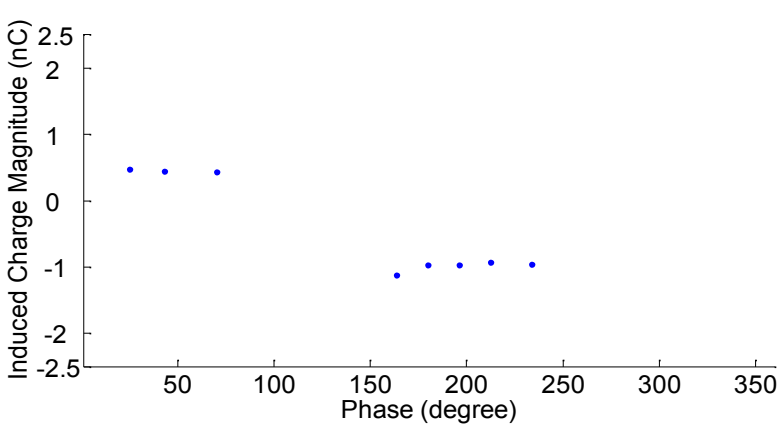

(a) $\tau=1 \mathrm{~ms}\left(N_{P D}=0.04\right)$

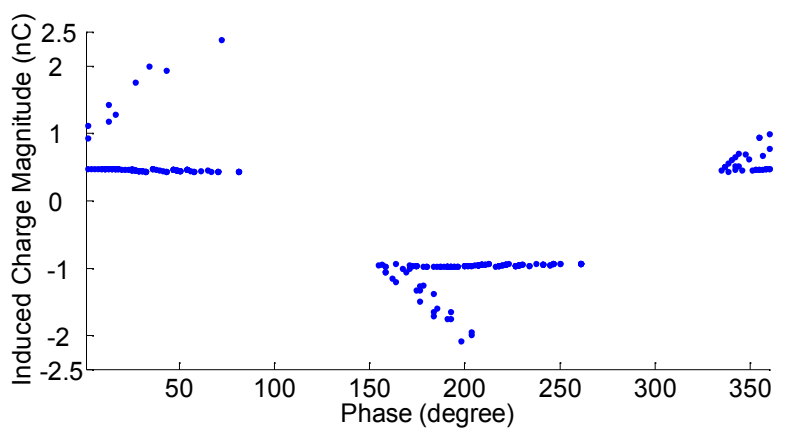

(b) $\tau=1.5 \mathrm{~ms}\left(N_{P D}=1.255\right)$

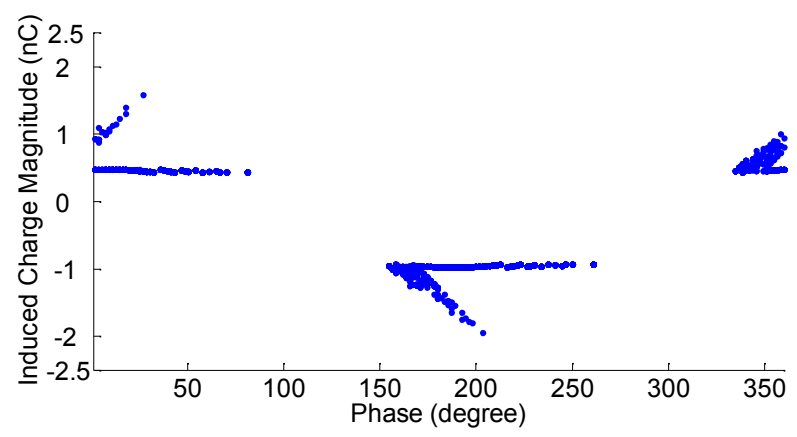

(c) $\tau=2 \mathrm{~ms}\left(N_{P D}=10.0\right)$

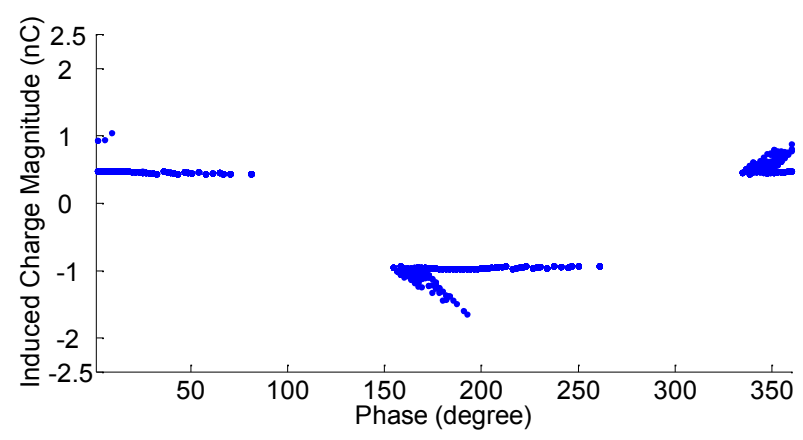

(d) $\tau=2.5 \mathrm{~ms}\left(N_{P D}=10.385\right)$

Figure 3. PRPD patterns for $\Phi=1.1 \mathrm{eV}$ and various $\tau$ under sinusoidal applied voltage
Figure 4 shows the plot of electric field as a function of time of the damped AC applied voltage. The curve is similar to Figure 2, except that the maximum applied voltage amplitude decreases with time.

The PRPD patterns under damped AC sinusoidal applied voltage when the decay constant, $\tau_{d}$ is varied are shown in Figure 5. When the decay constant of the damped $\mathrm{AC}$ waveform is increased, the number of PD per cycle, $N_{P D}$ decreases. This is because the magnitude of the electric field within the void decreases faster with time, which can be seen in the figure of the electric field plot as a function of time. Thus, the magnitude of the electric field in the void becomes less than the inception field at earlier time, preventing any PD from reoccurring. It can also be seen that the number of PDs found at the 'curvy' shape of the PRPD patterns under damped AC voltage is lower when the decay constant increases. In general, the pattern of PD activity under damped AC sinusoidal voltage is similar under the applied sinusoidal voltage. However, the 'curvy' shape of the PRPD pattern becomes less obvious when the decay constant is higher. The maximum PD charge magnitude also decreases under higher decay constant of the damped AC applied voltage.

\section{Impulse applied voltage}

Separated double exponential function can be used to represent an impulse voltage, which is represented by

$$
U=U_{\text {app }}\left[\exp \left(-\left(t-t_{0}\right) / \tau_{2}\right)-\exp \left(-\left(t-t_{0}\right) / \tau_{1}\right)\right]
$$

where $\tau_{1}$ and $\tau_{2}$ are the time constant and $t_{0}$ is the true origin of the impulse.

The model geometry and parameters that have been used for simulation of PD activity under impulse voltage is the same as in Figure 1 and Table 1. Figure 6 shows the electric field magnitude plot as a function of time of the impulse voltage, The applied voltage, $U_{a p p}$ is $50 \mathrm{kV}, t_{0}$ is zero and $\tau_{1}$ and $\tau_{2}$ are 1.2 us and 50 us. PDs can be seen to occur very frequently at the front time of the impulse voltage but less frequent at the tail time.

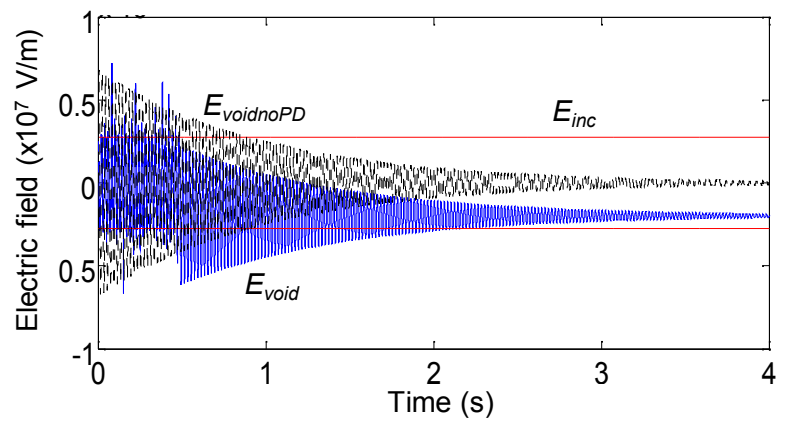

Figure 4. Electric field magnitude as a function of time of a damped AC sinusoidal applied voltage 


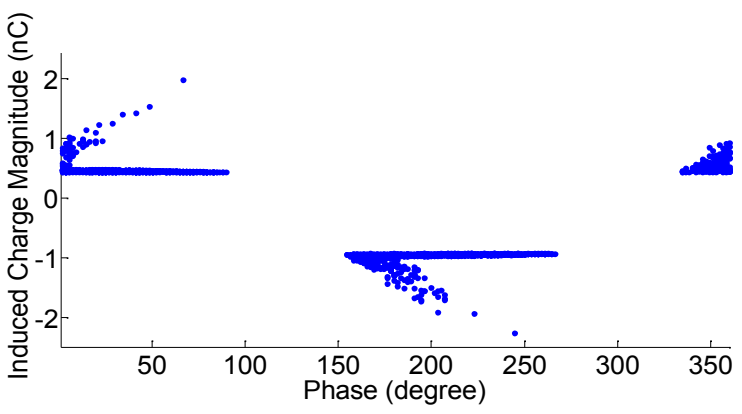

(a) Decay constant, $\tau_{d}=0.25\left(N_{P D}=8.415\right)$

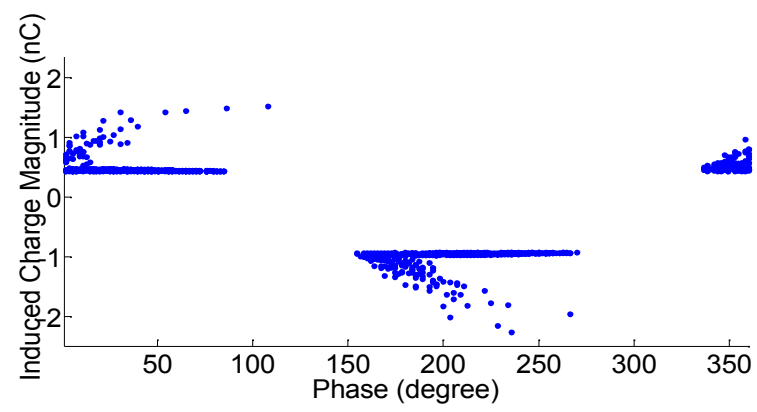

(b) Decay constant, $\tau_{d}=0.5\left(N_{P D}=4.965\right)$

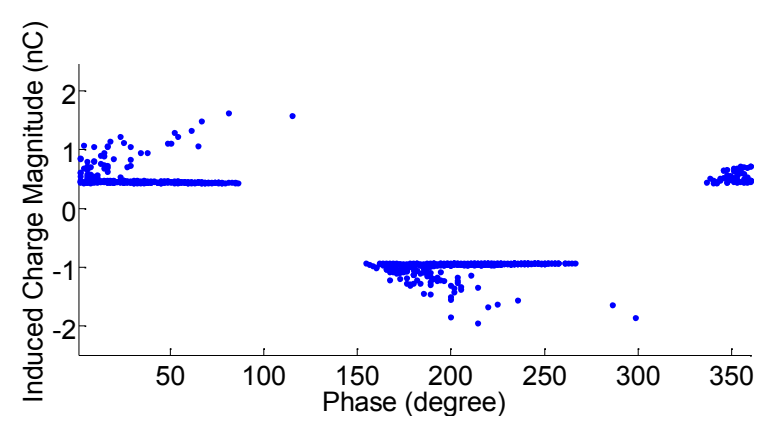

(c) Decay constant, $\tau_{d}=1\left(N_{P D}=2.86\right)$

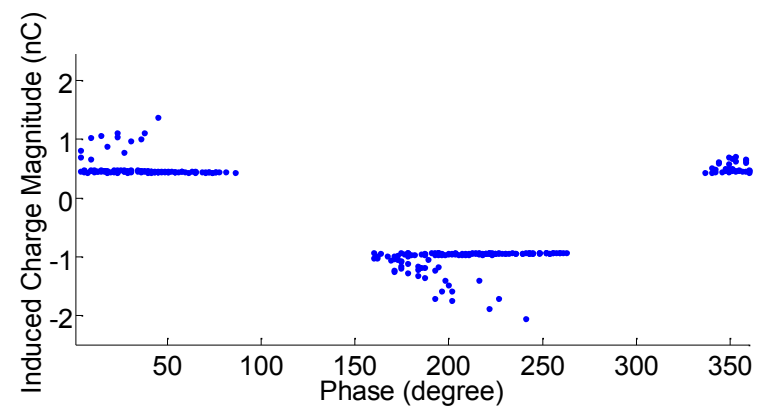

(d) Decay constant, $\tau_{d}=2\left(N_{P D}=1.68\right)$

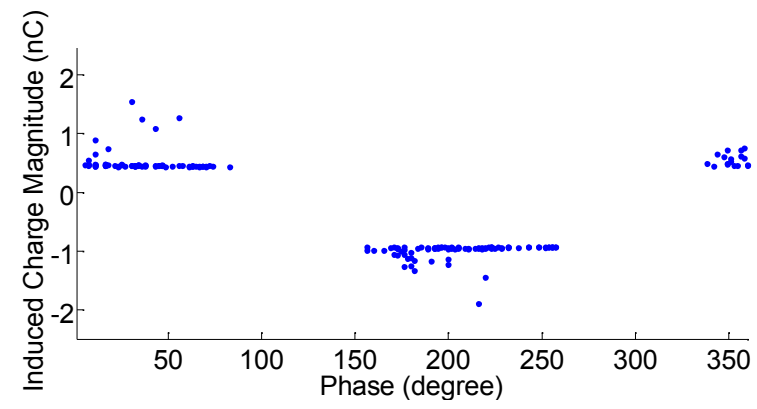

(e) Decay constant, $\tau_{d}=4(\mathrm{NPD}=0.945)$

Figure 5. PRPD patterns for different decay constant under damped AC sinusoidal applied voltage

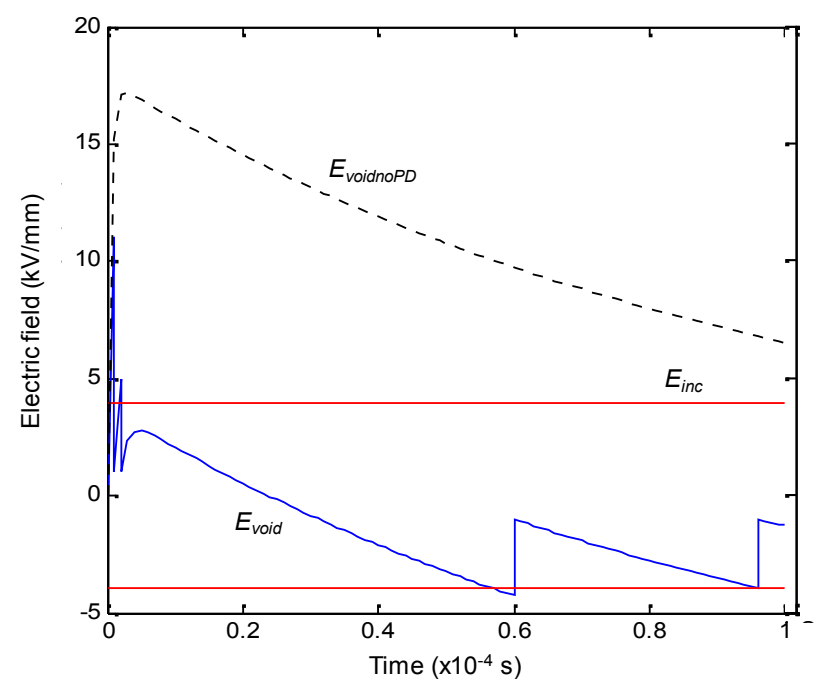

Figure 6. Electric field magnitude as a function of time of an impuse voltage

To observe PD behaviour under impulse applied voltage, the amplitude of the voltage is fixed at $300 \mathrm{kV}$ but the front time of the impulse voltage is varied. When the front time of the impulse voltage increases, the number of PDs within the first 100 us increases but the total number of PDs is almost the same. Under a longer front time, the impulse voltage increases slower. Thus, the probability of getting an initial free electron to initiate a PD is higher. This is due to longer time duration for the electric field magnitude in the void to reach the inception field. Therefore, more PDs can occur during the slower rise time of the impulse voltage. From the PRPD patterns, more PDs can be seen occurring when the front time of the impulse voltage is larger. 


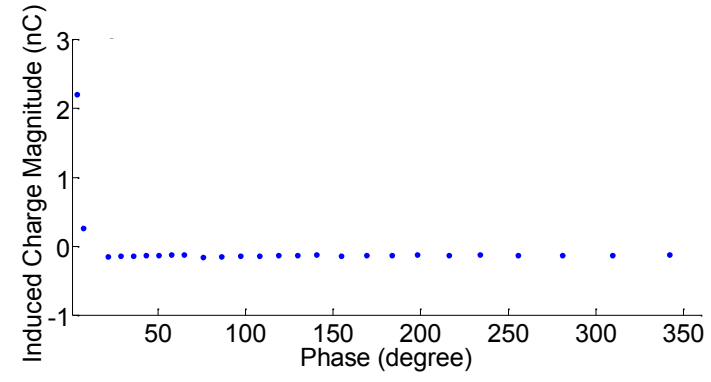

(a) Front time $=2$ us $\left(N_{P D}=12\right)$

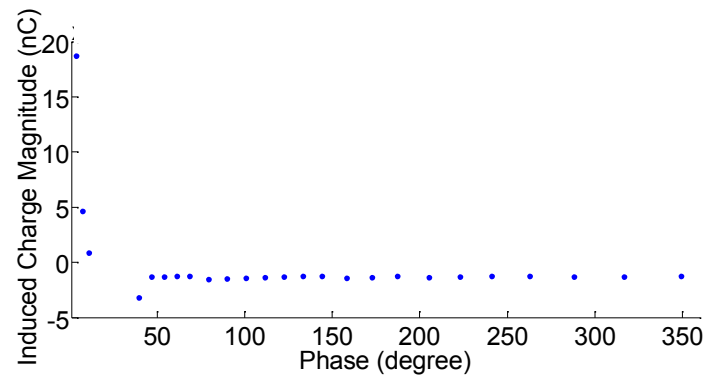

(b) Front time $=3$ us $\left(N_{P D}=12\right)$

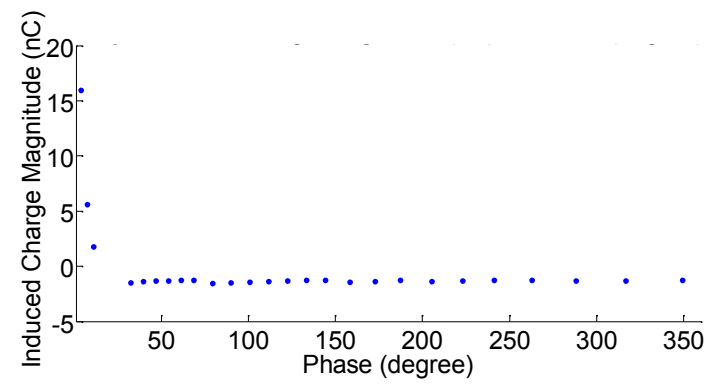

(c) Front time $=4$ us $\left(N_{P D}=12\right)$

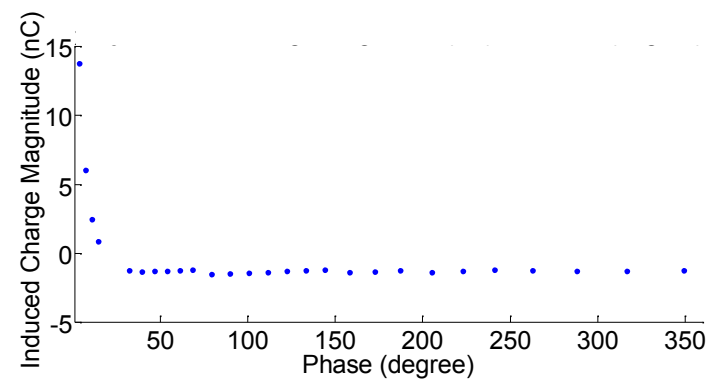

(d) Front time $=5$ us $\left(N_{P D}=13\right)$

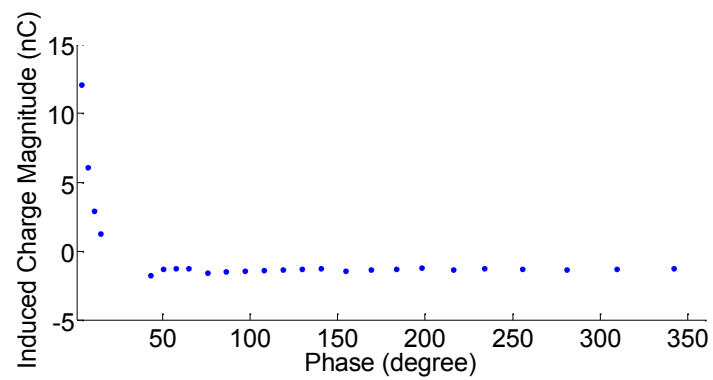

(e) Front time $=6$ us $\left(N_{P D}=13\right)$

Figure 7. PRPD patterns under different front time of the impulse voltage

\section{CONCLUSIONS}

Partial discharge (PD) behaviour under different applied voltages has been studied through simulation model in this work. PD behaviour was observed through phase resolved partial discharge (PRPD) patterns under AC sinusoidal, damped AC sinusoidal and impulse applied voltages. PD activity within a void in a dielectric material is strongly influenced by the applied voltage waveform because the electric field magnitude in the void is determined by the applied voltage. The maximum electric field in the void was calculated through a model geometry that has been developed using finite element analysis (FEA).

For PD activity under AC sinusoidal applied voltage, when the effective charge decay time constant increases, the number of PDs per cycle was found to increase. When the decay constant of the damped AC sinusoidal waveform increases, the number of PDs per cycle decreases. PRPD patterns changes with the change of the decay constant of the damped AC sinusoidal applied voltage. The number of PDs occurring within the front time of an applied impulse voltage increases when the front time is longer.

Future work will consider the actual experiment of PD activity under different applied voltage waveform. The measurement results will then be compared with the simulation results to identify critical parameters affecting PD activity under various types of the applied voltage.

\section{ACKNOWLEDGMENT}

The author thanks the University of Malaya for supporting this work through the HIR research grant (Grant no: H-1600100-D000048).

\section{REFERENCES}

[1] E. Kuffel, W. S. Zaengl, and J. Kuffel, High Voltage Engineering: Fundamentals, 2nd ed.: Newnes, Butterworth-Heinemann, 2000.

[2] H. Illias, G. Chen, and P. L. Lewin, "Partial Discharge within a Spherical Cavity in a Dielectric Material as a Function of Cavity Size and Material Temperature," IET Science, Measurement \& Technology, pp. 1-8, 2012.

[3] H. Illias, G. Chen, and P. L. Lewin, "The influence of spherical cavity surface charge distribution on the sequence of partial discharge events," Journal of Physics D: Applied Physics, vol. 44, pp. 1-15, 2011.

[4] H. Illias, G. Chen, and P. L. Lewin, "Modeling of partial discharge activity in spherical cavities within a dielectric material," IEEE Electrical Insulation Magazine, vol. 27, pp. 38-45, 2011.

[5] H. Illias, G. Chen, and P. L. Lewin, "Partial Discharge Behavior within a Spherical Cavity in a Solid Dielectric Material as a Function of Frequency and Amplitude of the Applied Voltage," IEEE Transactions on Dielectrics and Electrical Insulation, vol. 18, pp. 432-443, 2011.

[6] T. Do, O. Lesaint, and J. L. Auge, "Streamers and partial discharge mechanisms in silicone gel under impulse and AC voltages," IEEE Transactions on Dielectrics and Electrical Insulation, vol. 15, pp. 1526$1534,2008$.

[7] L. Niemeyer, "A generalized approach to partial discharge modeling," IEEE Transactions on Dielectrics and Electrical Insulation, vol. 2, pp. 510-528, 1995.

[8] F. Gutfleisch and L. Niemeyer, "Measurement and simulation of PD in epoxy voids," IEEE Transactions on Dielectrics and Electrical Insulation, vol. 2, pp. 729-743, 1995. 